\title{
SURVEILLANCE OF BACTERIA METHICILLIN-RESISTANT STAPHYLOCOCCUS AUREUS AND PSEUDOMONAS AERUGINOSA IN PATIENTS ADMITTED TO ORTHOPEDIC DEPARTMENT IN A TERTIARY REFERRAL HOSPITAL
}

\author{
PULIN BIHARI DAS ${ }^{1 *}$, MONALI PRIYADARSHINI MISHRA², SIBA NARAYAN RATH ${ }^{2}$
}

${ }^{1}$ Department of Orthopedics, Institute of Medical Sciences and Sum Hospital, Siksha 'O' Anusandhan University, K-8, Kalinga Nagar, Bhubaneswar - 751 003, Odisha, India. ' Central Research Laboratory, Institute of Medical Sciences and Sum Hospital, Siksha '0' Anusandhan University, K-8, Kalinga Nagar, Bhubaneswar - 751 003, Odisha, India. Email: pulin_bdas@yahoo.ca

Received: 06 December 2017, Revised and Accepted: 12 March 2018

ABSTRACT

Objective: Methicillin-resistant Staphylococcus aureus (MRSA) strains have emerged independently in diverse geographic zones and MRSA and Pseudomonas aeruginosa cause surgical site infections. Nosocomial surveillance in orthopedic surgery wards of the hospital for 16 months is presented.

Methods: A total of 621 wound swabs were cultured on blood and MacConkey agar plates for bacteria and Sabouraud dextrose agar for fungi.

Results: From 468 bacterial colonies, 98 MRSA and 74 P. aeruginosa strains and 41 fungal strains were isolated, and fungal strains were 13 strains of Aspergillus niger, and 28 strains of Candida albicans. P. aeruginosa and S. aureus strains were susceptible to antibiotics tobramycin, ciprofloxacin, piperacillin, vancomycin, levofloxacin, and amoxyclav. Similarly, A. niger and C. albicans were susceptible to antifungals, amphotericin B (AMB), liposomal AMB, itraconazole, voriconazole, posaconazole, and caspofungin.

Conclusion: Isolated MRSA strains were resistant to presently used common antibiotics, which attribute to the leading causatives of post-operative infection in orthopedic wounds, specifically.

Keywords: Surgical site infections, Hospital-acquired infection, Methicillin-resistant Staphylococcus aureus, Pseudomonas aeruginosa.

(c) 2018 The Authors. Published by Innovare Academic Sciences Pvt Ltd. This is an open access article under the CC BY license (http://creativecommons. org/licenses/by/4. 0/) DOI: http://dx.doi.org/10.22159/ajpcr.2018.v11i6.24136

\section{INTRODUCTION}

An acquired infection in hospital stay by a patient admitted for any health issue is defined as nosocomial or hospital-acquired infection (HAI) [1]. In general, HAIs are involved with surgical wounds, infection of urinary, respiratory tracts and soft tissues, giving rise to bloodstream infection (BSI), when uncontrolled. Those might be grave enough to surgical site infections (SSIs), leading to morbidity, functional disability, emotional suffering, longer hospitalization, and mortality [2-4]. These constitute major public health problems, promoting unwanted issues of "disability-adjusted life years." Moreover, SSIs accounting to $17 \%$ healthcare-associated infections are the second most common HAIs, next to urinary tract infection [5]. Indeed in orthopedic SSIs, cases of trauma, emergency surgery including dirty wound redressal were associated, and the later was a significant predictor of SSIs [6]. Other causes of SSIs have been identified as the lack of personal cleanliness of patients and indurate attitude of surgeons and paramedical staff toward stringent antiseptic maintenance of the total environment in operation theaters basically. Moreover, device associated [7] and hand washing and associated impeccable habits in maintaining basic hygiene. HAIs are often the underlying cause [8]. However, prophylactic antibiotics are given at the proper time at correct strength/dose and the use of clean surgical clothing or the check of flow of staff into operating room contribute to lowering the incidence of infections [9].

The commensal turned bacterium, Staphylococcus aureus is most prevalent in orthopedic SSIs. Particularly, several clonal variants of S. aureus were resistant to the penicillin group of antibiotics, after which methicillin/oxacillin was introduced for the control. Subsequently, methicillin-resistant $S$. aureus (MRSA), causing SSIs with/without the emergence of wounds [10]. The most gruesome situation is emergence of MRSA strains with concomitant resistance to most commonly used antibiotics of groups, aminoglycosides, macrolides, fluoroquinolones, chloramphenicol, tetracycline, cephalosporins and other $\beta$-lactams, amoxicillin-clavulanic acid, piperacillin-tazobactam carbapenem, and imipenem, as SSIs.

Moreover, Pseudomonas aeruginosa had been seen as a notorious pathogen in this hospital too [11]. These pathogens are mainly found in wounds and urinary tract but lead to innards causing septicemia and associated comorbidities, through BSI. As it is, the rate of invasion of a pathogenic bacterium directly depends on the level of drug resistance, apart from the challenged immune condition of patients.

This work describes surveillance of bacterial flora from wound sample of patients attending the orthopedic department of the hospital, over a period of 18 months. Two fungi, Aspergillus niger and Candida albicans were isolated along with bacteria. This surveillance was undertaken for a revision of the antimicrobial stewardship program; the rising concern from frequent SSIs reports in patients attending the orthopedic department with a newer prophylaxis module. Revised antimicrobial stewardship program would reduce nosocomial spread of virulent strains of bacteria, as well as morbidity including the cost of hospitalization.

\section{METHODS}

The present study was done between August 2015 and January 2017 in this hospital. Inclusion criteria were all closed fractures admitted to orthopedics department in this hospital. A total of 621 swabs were taken from the surgical site after definitive treatment of the fracture. Swabs were cultured on blood and MacConkey agar plates that were 
incubated at $37^{\circ} \mathrm{C}$ overnight for the growth of pathogenic bacteria, which were identified according to the standard method used for bacteria and concomitantly for fungi (Figs. 1 and 2). Antibiotic susceptibility tests of isolated bacteria were done according to Clinical Laboratory Standard Institute guidelines, as described by Mishra et al. and Rath et al. [12,13]. Standard antimicrobial discs (HiMedia, Mumbai) used for $S$. aureus were amikacin, amoxyclav, chloramphenicol, ciprofloxacin, cotrimoxazole, gentamicin, levofloxacin, linezolid, oxacillin, and vancomycin. Antimicrobial discs used for P. aeruginosa were amikacin, amoxyclav, ceftriaxone, ciprofloxacin, ceftazidime, gentamicin, piperacillin, netilmicin, ofloxacin, and tobramycin.

\section{Antibiotic sensitivity and detection of MRSA}

The standard MTCC number 7443 strain and all the isolated S. aureus strains were subjected to antibiotic sensitivity tests with antibiotics, by the Kirby-Bauer method (disc diffusion) detailed previously.

\section{Identification of fungi}

Direct microscopic examination of cotton swabs with samples was carried out by mounting sample lots treated with 1-2 drops of 1020\% KOH for 15-30 min. Each specimen was inoculated on two sets of Sabouraud dextrose agar slopes, one set with chloramphenicol, and the other set with cycloheximide (chloramphenicol - $0.05 \mathrm{mg} / \mathrm{mL}$ and cycloheximide $-0.5 \mathrm{mg} / \mathrm{mL}$ ). Cultures were incubated at room temperature for 4-6 weeks and were observed regularly for possible growth. Fungal isolates were identified on the basis of duration of growth and surface morphology of colonies, as well as pigment production on the reverse and microscopic examination of hyphae in lacto phenol cotton blue preparation [13].

\section{RESULTS}

From 621 collected samples, 509 bacterial and fungal colonies grew on agar plates, and no microbial growth was seen with 112 samples. There were 468 bacterial and 41 fungal isolates in total. The most common causal bacteria isolated were 250 isolates of $S$. aureus with and 74 isolates of $P$. aeruginosa; and 98 isolates of $S$. aureus were MRSA. Of 509 samples, isolated bacteria were in decreasing order (with number of isolated strains): Staphylococcus aureus (250) > P. aeruginosa (74) $>$ Acinetobacter baumannii (48) > Escherichia coli (24) > Klebsiella pneumoniae (20)> Enterobacter aerogenes (18) > Proteus vulgaris (15) $>$ Citrobacter sp. (10) > Enterococcus faecalis (09). Fungi accounted for 13 isolates of $A$. niger and 28 isolates of $C$. albicans from 509 growthyielding samples (Table1).

Antibiograms of the most common bacteria, P. aeruginosa and S. aureus (other than MRSA) were presented. The susceptibility rate of P. aeruginosa to tobramycin $10 \mu \mathrm{g} /$ disk had $91 \%$, followed by ciprofloxacin $5 \mu \mathrm{g} /$ disk $79 \%$ and piperacillin $100 \mu \mathrm{g} /$ disk $77 \%$ and $100 \%$ S. aureus isolates were susceptible to vancomycin $30 \mu \mathrm{g} /$ disk, followed by $88 \%$ to levofloxacin $5 \mu \mathrm{g} /$ disk and $77 \%$ isolates to amoxyclav $30 \mu \mathrm{g} /$ disk. Thus, all isolated strains of MRSA were multidrug resistant (MDR) (Fig. 3). With a cohort of 98 MRSA strains, the minimum inhibitory concentration (MIC) range against oxacillin was $16-512 \mu \mathrm{g} / \mathrm{mL}$, the MIC range of methicillinsensitive $S$. aureus was $1-4 \mu \mathrm{g} / \mathrm{mL}$. These MIC values confirmed the presence of MRSA strains, as the breakpoint for being resistant to oxacillin was $\geq 4 \mu \mathrm{g} / \mathrm{mL}$ (Table 2 and 3 ).

The antifungal susceptibility rate of A. niger to amphotericin B (AMB) was $82 \%$, followed by liposomal AMB $75 \%$ and itraconazole (ITC) $63 \%$, voriconazole (VRC) 55\%, posaconazole (POS) 48\%, and caspofungin (CPF) $32 \%$; similarly, susceptibility rate of C. albicans to AMB was $86 \%$, followed by liposomal AMB 77\% and ITC 69\%, VRC 62\%, POS 57\%, and CPF 49\% resistance (Fig. 4, Table 4).

\section{DISCUSSION}

MDR strains of MRSA and P. aeruginosa had emerged nosocomially, as post-operative infection in orthopedic surgery patients. Obviously, the nosocomial emergence of MDR strains of bacteria is basically associated with substantial morbidity, increased the length of hospital stay and
Table 1: Growth of bacteria in cultures of wound swabs of patients admitted to orthopedic wards

\begin{tabular}{lll}
\hline Organisms & $\begin{array}{l}\text { MTCC strain } \\
\text { number }\end{array}$ & $\begin{array}{l}\text { Total isolates } \\
\mathbf{n = 5 0 9}(\mathbf{1 0 0})\end{array}$ \\
\hline Enterococcus sp. & 439 & $09(01.76)$ \\
MRSA & & $98(19.25)$ \\
MSSA & 7443 & $152(29.86)$ \\
A. baumannii & 1425 & $48(09.43)$ \\
Citrobacter sp. & 1658 & $10(01.96)$ \\
E. aerogenes & 2990 & $18(03.53)$ \\
E. coli & 443 & $24(04.71)$ \\
Klebsiella sp. & 2275 & $20(03.92)$ \\
P. vulgaris & 1771 & $15(02.94)$ \\
P. aeruginosa & 1688 & $74(14.53)$ \\
A. niger & 872 & $13(02.55)$ \\
C. albicans & 1425 & $28(05.50)$ \\
\hline
\end{tabular}

MRSA: Methicillin-resistant Staphylococcus aureus, MSSA: Methicillin-sensitive Staphylococcus aureus, the standard strain, percent values are in parenthesis, $\mathrm{n}$ or total isolates $=509$, from the total 621 samples; the rest 112 samples had no growth. P. aeruginosa: Pseudomonas aeruginosa, A. niger: Aspergillus niger, E. coli: Escherichia coli, E. aerogenes: Enterobacter aerogenes, P. vulgaris: Proteus vulgaris, A. baumannii: Acinetobacter baumannii, C. albicans: Candida albicans

Table 2: Antibiogram of resistance S. aureus and P. aeruginosa

\begin{tabular}{lll}
\hline Antibiotics & S. aureus & P. aeruginosa \\
\hline $\mathrm{Ac}$ & 39 & 26 \\
$\mathrm{Ak}$ & 23 & 28 \\
$\mathrm{Cf}$ & $\mathrm{Nd}$ & 24 \\
$\mathrm{Ch}$ & 29 & $\mathrm{Nd}$ \\
$\mathrm{Cot}$ & 34 & $\mathrm{Nd}$ \\
$\mathrm{Cp}$ & 38 & 21 \\
$\mathrm{Cz}$ & $\mathrm{Nd}$ & 32 \\
$\mathrm{Ge}$ & 25 & 36 \\
$\mathrm{Le}$ & 12 & $\mathrm{Nd}$ \\
$\mathrm{Lz}$ & 32 & $\mathrm{Nd}$ \\
$\mathrm{Ne}$ & $\mathrm{Nd}$ & 25 \\
$\mathrm{Of}$ & $\mathrm{Nd}$ & 35 \\
$\mathrm{Ox}$ & 42 & $\mathrm{Nd}$ \\
$\mathrm{Pi}$ & $\mathrm{Nd}$ & 23 \\
$\mathrm{~Tb}$ & $\mathrm{Nd}$ & 09 \\
$\mathrm{~V}$ & 0 & $\mathrm{Nd}$
\end{tabular}

Antibiotic in $\mu \mathrm{g} /$ disc: Ac: Amikacin 30, Ak: Amoxyclav 30, Cf: Ceftriaxone 30, Ch: Chloramphenicol 30, Cp: Ciprofloxacin 5, Cot: Cotrimoxazole 25, Cz: Ceftazidime 30, Ge: Gentamicin 10, Le: Levofloxacin 5, Lz: Linezolid 30, Ne: Netilmicin 30, Of: Ofloxacin 5, Ox: Oxacillin 1, Pi: Piperacillin 100, Tb: Tobramycin 10, V: Vancomycin 30, Nd: Not done. P. aeruginosa: Pseudomonas aeruginosa, S. aureus: Staphylococcus aureus

Table 3: Detection of MRSA and MSSA isolates based on MIC values from the presence of oxacillin in $12 \times 8 \mu$ l plates

\begin{tabular}{llll}
\hline Well & Oxacillin $(\boldsymbol{\mu g} / \mathbf{m L})$ & \multicolumn{2}{c}{ Number of isolates } \\
\cline { 3 - 4 } & & MRSA=98 & MSSA=152 \\
\hline 1 & 0 & 98 & 152 \\
2 & $\leq 0.25$ & - & - \\
3 & 0.5 & - & - \\
4 & 1 & - & 68 \\
5 & 2 & - & 36 \\
6 & 4 & - & 48 \\
7 & 8 & - & - \\
8 & 16 & 12 & - \\
9 & 32 & 18 & - \\
10 & 64 & 20 & - \\
11 & 128 & 22 & - \\
12 & $\geq 256$ & 26 & - \\
\hline
\end{tabular}

The oxacillin stock solution, $512 \mu \mathrm{g} / \mathrm{mL}$ was serially diluted at each successive well, from the $12^{\text {th }}$ well for a final concentration of $0.25 \mu \mathrm{g} / \mathrm{mL}$ oxacillin at the $2^{\text {nd }}$ well was obtained; -, no growth. MRSA: Methicillin-resistant Staphylococcus aureus, MSSA: Methicillin-sensitive Staphylococcus aureus, MIC: Minimum inhibitory concentration 
Table 4: Antifungal agents used against $A$. niger and $C$. albicans

\begin{tabular}{lll}
\hline Antifungal & A. niger & C. albicans \\
\hline AMB & 82 & 86 \\
1- AMB & 75 & 77 \\
ITC & 63 & 69 \\
VRC & 55 & 62 \\
POS & 48 & 57 \\
CPF & 32 & 49 \\
\hline
\end{tabular}

Antifungal agents: AMB: Amphotericin B, l- AMB: Liposomal AMB, ITC: Itraconazole, VRC: Voriconazole, POS: Posaconazole, CPF: Caspofungin. A. niger: Aspergillus niger, C. albicans: Candida albicans

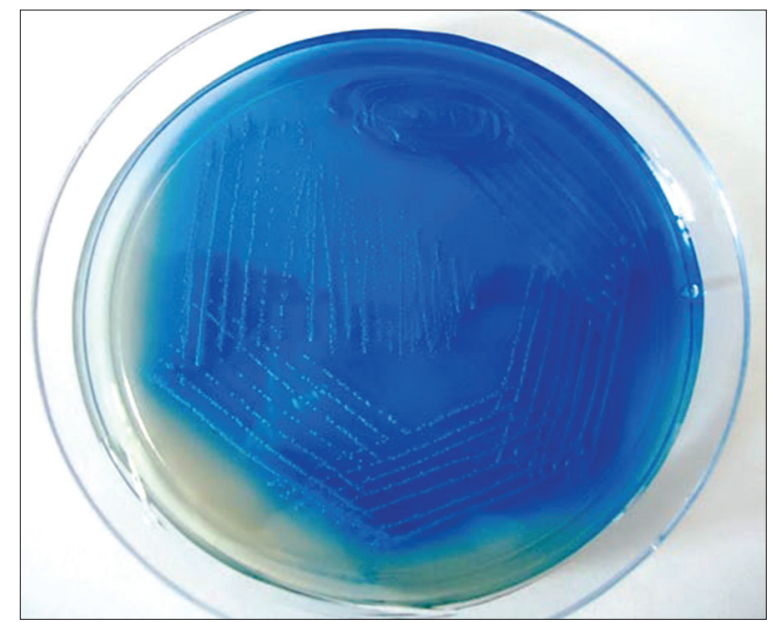

Fig. 1: Methicillin-resistant Staphylococcus aureus on MeReSa chromogenic agar

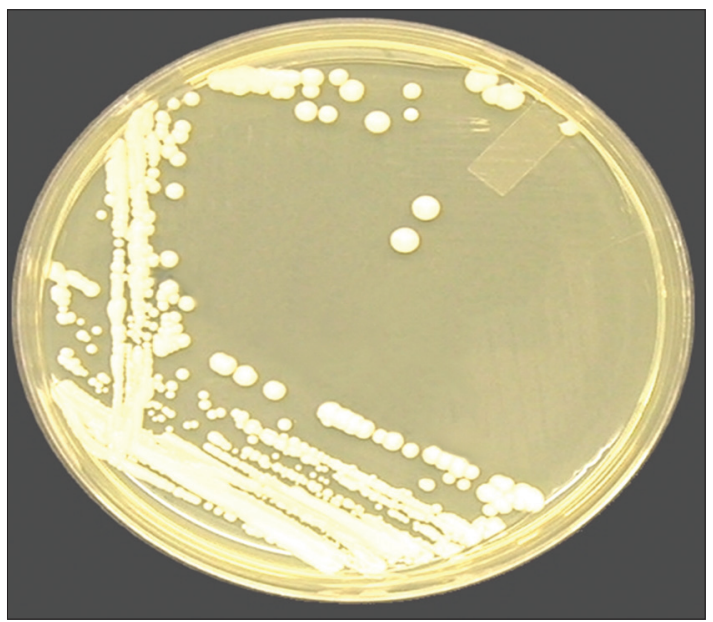

Fig. 2: Candida albicans in potato dextrose agar media

a higher incidence of amputation and graft removal, particularly in orthopedic surgery patients. Thus, greater emphasis on pre-operative screening protocols for colonization of these pathogens should be considered, accordingly for infection control measures aggressively, with minor alteration of pre-operative prophylactic antimicrobial uses; and meticulous post-operative surveillance for MRSA infection is a dire necessity for this superbug of health domain. Antimicrobial treatment should include empiric coverage for MRSA in institutions where MRSA is endemic. It was found that in a study from Serbia, S. aureus was recorded as the most frequently isolated pathogen from SSIs isolated pathogens of which $43.7 \%$ were MRSA; $81.5 \%$ P. aeruginosa strains were resistant to fluoroquinolones and carbapenems [14]. A prolonged pre-operative hospital stay with exposure to a hospital environment had been shown to increase the risk from SSI wound contamination [15]. It was also reported a higher rate of SSI in patients with a prolonged pre-operative hospital stay. Indeed, prolonged pre-operative hospital stay leads to colonization with antimicrobial resistant microorganisms by providing increased opportunity for ultimate bacterial colonization [16]. Eventually, this may to lead septicemia/bacteremia that may to lead to amputation in the absence of emulating control required for MDR bacteria.

In the present study, $S$. aureus was predominant in surgical sites, followed by P. aeruginosa and Klebsiella sp., while, E. coli, Citrobacter, and Proteus sp. were too isolated from surgical sites, corroborating another report [17]. Many studies have reported $S$. aureus as the most common isolate from the post-operative wound infection [18]. Furthermore, the incidence of isolated Gram-negative bacteria in surgical wounds can be attributed to be acquired from patient's normal endogenous microflora [18].

In 5 years study from Saudi Arabia, of total 830 patients, 29.11\% MRSA, 21.5\% Acinetobacter sp., 18.9\% Pseudomonas sp., and 17.7\% Enterococcus sp. were recorded. Emergency surgical procedures carried the greatest risk with Staphylococcus sp. and Acinetobacter sp. being the most common infecting bacteria from treatments of dirty wounds. Similar to MRSA, methicillin-resistant Staphylococcus epidermidis strains were reportedly frequently nosocomially in orthopedic wards $[19,20]$. Resistant Gram-negative forms of bacteria were increasingly prevalent in hospitals and communities [20]. As known, tibial plateau fractures are challenging of treatment due to the high incidence of post-operative infections. A retrospective review was undertaken to identify all patients with tibial plateau fractures over a 10-year period (2003-2012), who underwent open reduction internal fixation. MRSA was the most common species [21]. This study demonstrated that most of these pathogens isolated from clinical samples were MDR, and those are potentially enough to destroy the clinical totem pole of a hospital and to precipitate devastating episodes in the community. As analyzed, suppurative infections are one of the major problems of health, as MDR bacteria could attack several organs such as lungs, heart, and kidneys, through BSI [22,23].

\section{CONCLUSION}

This surveillance was undertaken for a revision of the antimicrobial stewardship program especially for surgical episodes; the rising concern from frequent SSIs reports in patients attending the orthopedic department with a newer prophylaxis module. A revised antimicrobial stewardship program would reduce nosocomial spread of virulent strains of bacteria, as well as morbidity including the cost of hospitalization. MRSA and P. aeruginosa were leading causatives of postoperative infection in orthopedic wounds. Antimicrobial treatment should be revised in empiric coverage for surgical wounds, in view of shenanigans of both pathogens.

\section{ACKNOWLEDGMENTS}

This work, R.N. Padhy (RNP) is PI; M. P. Mishra and S. N. Rath were supported as JRFs by a major research project on "Development of standardized herbal extracts against urinary tract bacterial infection" (Grant no. BT/PR8214/PBD/17/863/2013), from the Department of Biotechnology, Government of India, New Delhi.

\section{AUTHORS CONTRIBUTION}

PBD, conducted the clinical study, MPM and SNR helped PBD in microbiological study, RNP directed the work holistically in which PBD and SNR wrote the draft copy of the paper. All authors approved the manuscript.

\section{CONFLICT OF INTEREST}

The authors have no conflict of interest. 


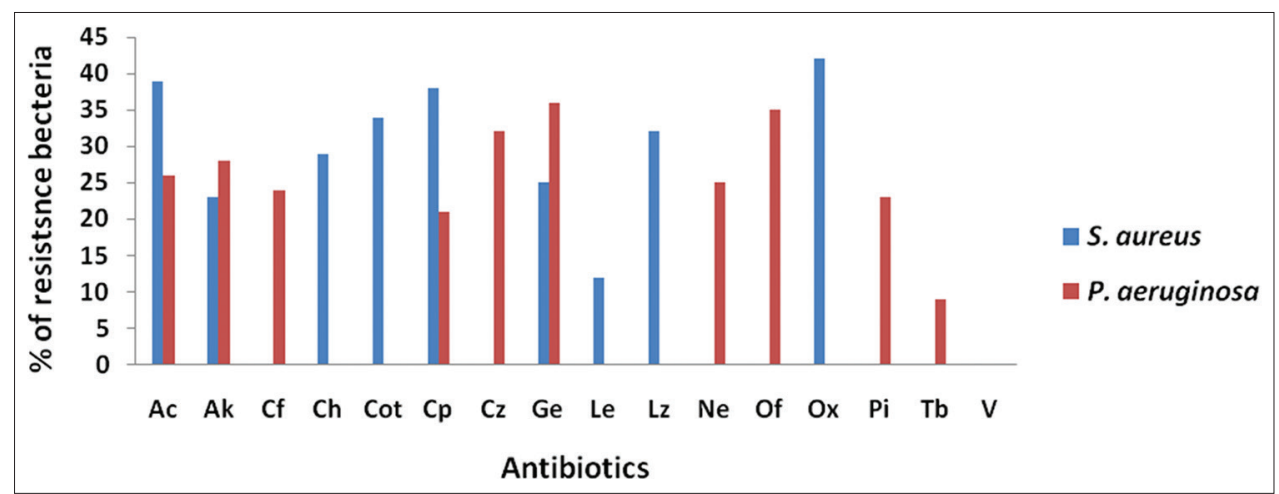

Fig. 3: Antibiogram of resistance bacteria, Pseudomonas aeruginosa and S. aureus. Antibiotic in $\mu \mathrm{g} / \mathrm{disc}$ : Ac: Amikacin 30, Ak: Amoxyclav 30, Cf: Ceftriaxone 30, Ch: chloramphenicol 30, Cp: Ciprofloxacin 5, Cot: Cotrimoxazole 25, Cz: Ceftazidime 30, Ge: Gentamicin 10, Le: Levofloxacin 5, Lz: Linezolid 30, Ne: Netilmicin 30, Of: Ofloxacin 5, Ox: Oxacillin 1, Pi: Piperacillin 100, Tb: Tobramycin 10, V: Vancomycin 30

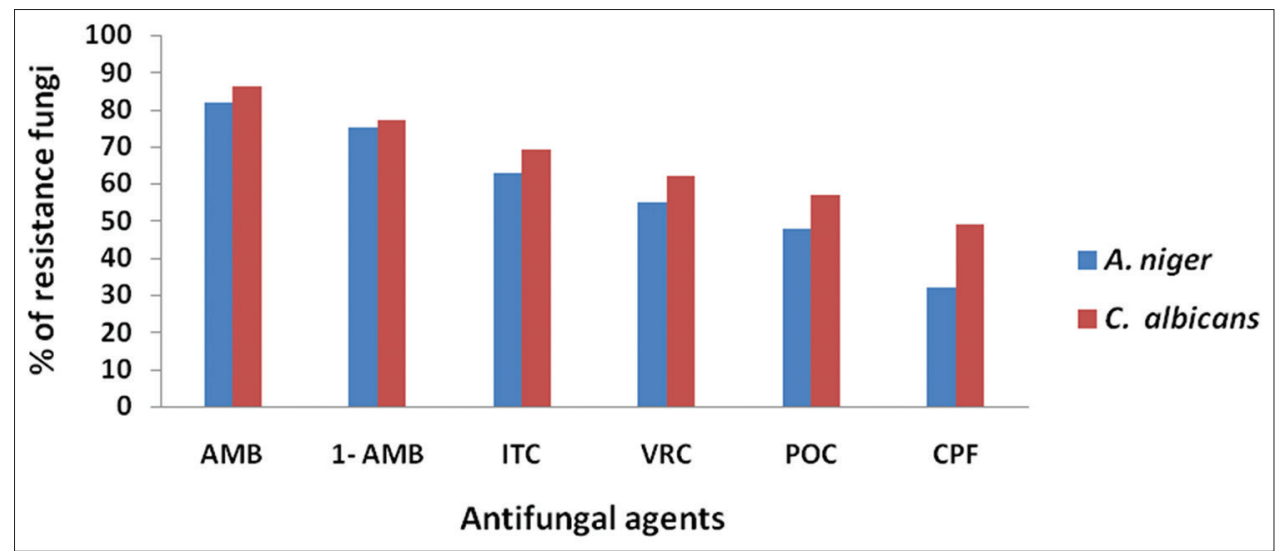

Fig. 4: Antifungal agents used against fungi, Aspergillus niger and Candida albicans. Antifungal agents: AMB: Amphotericin B; 1-AMB: Liposomal AMB, ITC: Itraconazole, VRC: Voriconazole, POS: Posaconazole, CPF: Caspofungin

\section{REFERENCES}

1. WHO. Prevention of Hospital Acquired Infections: A Practical Guide. Malta: Department of Communicable Disease, Surveillance and Response; 2002. Available from: http://www.who.int/csr/resources/ publications/whocdscsreph200212.pdf. [Last accessed on $2010 \mathrm{Jul}$ 20].

2. Endalafer N, Gebre-Selassie S, Kotisso B. Nosocomial bacterial infections in a tertiary hospital in Ethiopia. J Infect Prev 2011;12:38-43.

3. Napolitano MN. Perspectives in surgical infections: What does the Future hold? Surg Infect 2010; 11:111-23.

4. Datta R, Huang SS. Risk of infection and death due to methicillinresistant Staphylococcus aureus in long-term carriers. Clin Infect Dis 2008;47:176-81.

5. Wisplinghoff $H$, Bischoff $T$, Tallent SM, Seifert H, Wenzel RP, Edmond MB. Nosocomial bloodstream infections in US hospitals: Analysis of 24,179 cases from a prospective nationwide surveillance study. Clin Infect Dis 2004;39:309-17.

6. Nguyen D, MacLeod WB, Cam PD, Cong QT. Incidence and predictors of surgical-site infections in vietnam. Infect Control Hosp Epidemiol 2001;22:485-93.

7. Whitehouse JD, Friedman ND, Kirkland KB, Richardson WJ, Sexton DJ. The impact of surgical-site infections following orthopedic surgery at a community hospital and a university hospital: Adverse quality of life, excess length of stay, and extra cost. Infect Control Hosp Epidemiol 2002;23:183-9.

8. Pittet D. Compliance with hand disinfection and its impact on hospitalacquired infections. J Hosp Infect 2001;48 Suppl A: S40-6.

9. Tammelin A, Ljungqvist B, Reinm"uller B. Comparison of three distinct surgical clothing systems for protection from air-borne bacteria: a prospective observational study. Patient Saf Surg 2012;15:23.

10. Dubey D, Rath S, Sahu MC, Patnaik L, Debata NK, Padhy RN.
Surveillance of infection status of drug resistant Staphylococcus aureus in an Indian teaching hospital. Asian Pacif J Trop Dis 2013;3:133-42.

11. Rath S, Padhy RN. Surveillance of acute community acquired urinary tract bacterial infections. J Acute Dis 2015;3:186-95.

12. Mishra MP, Debata NK, Padhy RN. Surveillance of multidrug resistant uropathogenic bacteria in hospitalized patients in Indian. Asian Pac J Trop Biomed 2013;3:315-24

13. Rath S, Panda M, Sahu MC, Padhy RN. Bayesian analysis of two diagnostic methods for paediatric ringworm infections in a teaching hospital. J Mycol Med 2015;25:191-9.

14. Starčević S, Munitlak S, Mijović B, Mikić D, Suljagić V. Surgical site infection surveillance in orthopedic patients in the military medical academy, belgrade. Vojnosanit Pregl 2015;72:499-504.

15. Lilani SP, Jangale N, Chowdhary A, Daver GB. Surgical site infection in clean and clean-contaminated cases. Indian $\mathrm{J}$ Med Microbiol 2005;23:249-52

16. Patel SM, Patel MH, Patel SD, Soni ST, Kinariwala DM, Vegad MM. Surgical site infections: Incidence and risk factors in a tertiary care hospital, Western India. Nat J Community Med 2012;3:193-6.

17. Mahesh CB, Shivakumar S, Suresh BS, Chidanand SP, Vishwanath Y. A prospective study of surgical site infections in a teaching hospital. J Clin Diagn Res 2010;4:114-9.

18. Malik S, Gupta A, Singh KP, Agarwal J, Singh M. Antibiogram of aerobic bacterial isolates from post-operative wound infections at a tertiary care hospital in India. J Infect Dis Antimicrob Agents 2011;28:45-52

19. Al-Mulhim FA, Baragbah MA, Sadat-Ali M, Alomran AS, Azam MQ. Prevalence of surgical site infection in orthopedic surgery: A 5-year analysis. Int Surg 2014;99:264-8.

20. Uçkay I, Pittet D, Vaudaux P, Sax H, Lew D, Waldvogel F, et al. Foreign body infections due to Staphylococcus epidermidis. Ann Med 
2009;41:109-19.

21. Momaya AM, Hlavacek J, Etier B, Johannesmeyer D, Oladeji LO, Niemeier TE, et al. Risk factors for infection after operative fixation of tibial plateau fractures. Injury 2016;47:1501-5.

22. Techaoei S, Eakwaropas P, Khemjira J, Warachate K. Structure characterization and evaluation potential of antimicrobial. Extracts from
Phellinus linteus against skin infectious pathogens, Staphylococcus epidermidis atcc12228 and Propionibacterium acnes dmst14916. Int $J$ Pharm Sci 2017:9:70-81.

23. Penta J, Jannu K, Musthyala R. Antimicrobial studies of selected antibiotics and their combination with enzymes. Int $J$ Pharm Sci $2010 ; 2: 43-4$ 